\title{
Design of an optimal state derivative feedback LQR controller and its application to an offshore steel jacket platform
}

\author{
Hakan Yazici $^{a *}$ (D) and Mert Sever ${ }^{b}$ \\ ${ }^{a}$ Department of Mechanical Engineering, Yildiz TechnicalUniversity, Turkey \\ ${ }^{b}$ Department of Mechatronics Engineering, Yildiz Technical University, Turkey \\ hyazici@yildiz.edu.tr,msever@yildiz.edu.tr
}

\section{ARTICLE INFO}

Article history:

Received: 27 April 2017

Accepted: 24 October 2017

Available Online: 8 November 2017

\section{Keywords:}

Optimal control

State derivative feedback

Convex optimization

Offshore steel jacket platform

AMS Classification 2010:

49J15, $90 \mathrm{C} 22$

\begin{abstract}
This paper concerns with the optimal state derivative feedback LQR controller design for vibration control of an offshore steel jacket platform having active tuned mass damper against the wave induced disturbances. Considering that the state derivative signals such as acceleration and velocity are easier to measure rather than the state variables such as displacement, state derivative feedback control strategy is proposed to obtain practically applicable and easily realizable synthesis method. On the basis of convex optimization approach, state derivative feedback LQR controller design is formulated in Linear Matrix Inequalities (LMIs) form to get an optimal feasible solution set. Finally, an offshore steel jacket platform subject to nonlinear self excited wave force is used to illustrate the effectiveness of the proposed approach through simulations. The results show that proposed state derivative LQR controller is very effective in reducing vibration amplitudes of each floor of modeled offshore steel jacket platform and achieves compitable control performance to classical LQR controller design.
\end{abstract}

\section{Introduction}

The offshore steel jacket platforms play an important role in the oil exploration and drilling operation in the oceans [1]. As it is well known that offshore steel jacket paltforms are exposed to the various disturbances such as strong winds, ocean waves, earthquakes which are caused the structural vibrations and makes them very vulnerable and unsafe [2], [3]. Therefore, vibration control of offshore steel jacket platforms subject to environmental disturbances and working conditions has been receving a great deal of interest for the last two decades, and a lot of research effort has been devoted to the development of advanced control algorithms.

Abdel-Rohman has modeled a realistic offshore steal jacket platform using finite elements method to control the structural vibrations against wave loads [1]. Multiloop feedback controller design has been developed in Terro [2] et al., for nonlinear wave excited steel jacket platforms. Wu et al. have dealt with the non-fragile state feedback $\mathrm{H}_{\infty}$ control problem to attenuate vibrations of steel-jacket platform subject to regular wave disturbance [4]. Mei et al. have proposed the design of a fuzzy $\mathrm{H}_{\infty}$ controller for active vibration control of an offshore platform with parameter uncertainties [5]. Sliding mode $\mathrm{H}_{\infty}$ vibration control problem has been considered in Zhang et al [6], for offshore steal jacket platform having nonlinear self excited wave forces and external disturbances. Li et al., have applied state feedback $\mathrm{H}_{2}$ controller in reducing the effect of wave laoding on offshore platform [7]. State feedback stabilization control problem for offshore steel jacket platforms having actuator delay has been considered in Zhang et al [8]. They have assumed that all the state variables of the offshore platform are available for measurement. Zhang and Han have applied network based modelling and active vibration control for offshore steel jacket platform having tuned mass damper.

As it can be observed from the summarised literature, papers that address the state feedback vibration control problem of offshore steel jacket platforms are quite a few. As it is well known that state feedback controller assumes that all the state variables are available for measurement which are displacement and velocities in active structural control problem. However, displacement signals are not possible to be obtained accurately by integration, since the accelerometers are noisy and contain dc offset in low frequency region.

\footnotetext{
*Corresponding author
} 
Therefore, a pure integrator is not practical and should be combined with a high-pass-filter (HPF) to remove the integrator drift. An HPF with sufficiently large time constant, results in a phase error. It is apparently seen that considerable amount of effort is required to accurately integrate acceleration signals. In addition, computation accuracy will gradually decrease by getting displacement from acceleration with double step integration process [10]. In the light of aforementioned considerations, state derivative feedback controller design strategy is a promising active structural controller approach in the following aspects. Firstly, only a single step integration is needed to obtain velocity for accelerometer outputs. Note that accelerometers are one of the most common sensors in active structural control problems. Secondly, closed loop system order has not been increased, since the state derivative feedback controllers are static and memoryless with no additional state variables. This situation motivates us with the fact that there exist still more room obtaining practically applicable optimal state derivative feedback LQR controller synthesis to attenuate the vibrations of offshore platforms. Moreover, to the best of authors' knowledge it can be seen that there is no result has been given in the literature on the active vibration control of offshore steel jacket platform by the use of state derivative feedback approach, so far.

In this study, because of the state derivative signals are easier to measure, an LMI based optimal state derivative LQR controller is developed to control of the offshore steel jacket platform having nonlinear wave disturbances. In controller design, first, stability and solvability conditions of an optimal state derivative feedback LQR controller is presented in LMI form and minimization of quadratic cost function is ensured by the use of convex optimization techniques. Then, in order to compare the proposed method, well known classical LQR controller is designed. Last, numerical simulations studies have been conducted to illustrate the effectiveness of the propsed control staregy. The main importance of this study is to develop an easily realizable synthesis method to obtain practically applicable optimal state derivative LQR controller achieves comparable performance improvement with the conventional state feedback LQR controller.

Rest of papers organized as follows. Mathematical model of the realistic offshore steel jacket platform and formulations of nonlinear Morison Equations based wave force are given in Section 2. The design of proposed state derivative feedback LQR controller are presented in Section 3. Simulation resulst with discussions are given in Section 4. Finally, Section 5 concludes the paper.

Notation: The notation to be used in the paper is fairly standard. $\mathfrak{R}$ stands for the set of real numbers, $\mathfrak{R}^{n \times n}$ is the set of $n \times n$ dimensional real matrices. 'diag' denotes the diagonal matrices. The identity and null matrices are denoted by $I$ and 0 , respectively.
$X>0(\geq, \leq 0)$ denotes that $X$ is a positive definite (positive semi-definite, negative definite) matrix. The notation '*' denotes off-diagonal block completion of a symmetric matrix. Finally, $\operatorname{diag}\left\{M_{1}, \ldots, M_{n}\right\}$ stands for a diagonal matrix with elements $M_{l}, \ldots, M_{n}$ appearing on its diagonal.

\section{Mathematical modeling of offshore steel jacket platform}

In this section, a realistic offshore steel jacket platform model that includes an Active Tuned Mass Damper (ATMD) is used for controller design as shown in Figure 1 [2]. In this model, ATMD is used as an active control mechanism to supress structural vibrations, which is installed on the top floor of the offshore steel jacket platform.

The equations of the motion of the considered offshore steel jacket platform have been formulated in [2], [8], by the use of first two dominant vibration modes of the system as,

$$
\begin{aligned}
z_{1}(t)= & -2 \xi_{1} \omega_{1} z_{1}(t) \\
& -\phi_{1} C_{T}\left[\phi_{1} z_{1}(t)+\phi_{2} z_{2}(t)\right] \\
& +\phi_{1} C_{T} z_{T}(t)-\omega_{1}^{2} z_{1}(t) \\
& -\phi_{1} K_{T}\left[\phi_{1} z_{1}(t)+\phi_{2} z_{2}(t)\right] \\
& +\phi_{1} K_{T} z_{T}(t)-\phi_{1} u(t) \\
& \left.+f_{1}\left(z_{1}(t), z_{2}(t), t\right)\right) \\
& +f_{2}\left(z_{1}(t), z_{2}(t), t\right) \\
z_{2}(t) & =-2 \xi_{2} \omega_{2} z_{2}(t) \\
& -\phi_{2} C_{T}\left[\phi_{1} z_{1}(t)+\phi_{2} z_{2}(t)\right] \\
& +\phi_{2} C_{T} z_{T}(t)-\omega_{2}^{2} z_{2}(t) \\
& -\phi_{2} K_{T}\left[\phi_{1} z_{1}(t)+\phi_{2} z_{2}(t)\right] \\
& +\phi_{2} K_{T} z_{T}(t)-\phi_{2} u(t) \\
& +f_{3}\left(z_{1}(t), z_{2}(t), t\right) \\
& +f_{4}\left(z_{1}(t), z_{2}(t), t\right) \\
z_{T}(t)= & -2 \xi_{T} \omega_{T} z_{T}(t) \\
& +2 \xi_{T} \omega_{T}\left[\phi_{1} z_{1}(t)+\phi_{2} z_{2}(t)\right] \\
& +\frac{1}{m_{T}} u(t) \\
& +\omega_{T}^{2}\left[\phi_{1} z_{1}(t)+\phi_{2} z_{2}(t)\right] \\
& -\omega_{T}^{2} z_{T}(t) . \\
& z(t)
\end{aligned}
$$

Here, $z_{1}(t)$ and $z_{2}(t)$ are the generalized coordinates, which represent the first and second vibration modes of modeled offshore steel jacket platform, respectively. $\omega_{1}$ and $\omega_{2}$ are the natural frequencies of the first and second modes of vibration, repectively. $\xi_{1}$ and $\xi_{2}$ are damping ratios of the first and second modes of vibration, respectively; $\phi_{1}$ and $\phi_{2}$ are the first and second mode shapes, respectively. $z_{T}(t)$ represents the horizontal displacement of ATMD, $\xi_{T}$ and $\omega_{T}$ are the damping ratio and natural frequency of ATMD, respectively. $m_{T}$, 
$C_{T}$ and $K_{T}$ are mass, damping and stiffness of ATMD, respectively. $f_{1}, f_{2}, f_{3}$ and $f_{4}$ are the nonlinear wave force and $u(t)$ represents the active control force.

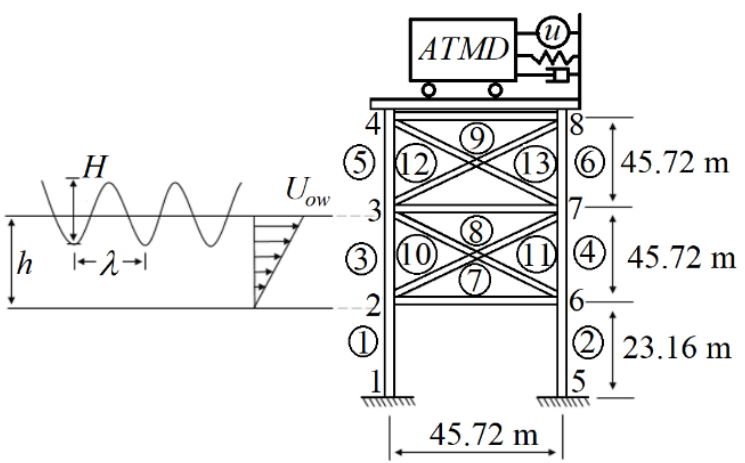

Figure 1. A simplified offshore steel jacket platform having ATMD [2].

The state variables and exogenous input can be defined as follow:

$$
\begin{gathered}
x(t):=\left[\begin{array}{lll}
x_{1}(t) & x_{2}(t) & x_{3}(t) \\
x_{4}(t) & x_{5}(t) & x_{6}(t)
\end{array}\right]^{T}, \\
w(x, t):=\left[\begin{array}{l}
f_{1}\left(x_{1}, x_{3}, t\right)+f_{2}\left(x_{1}, x_{3}, t\right) \\
f_{3}\left(x_{1}, x_{3}, t\right)+f_{4}\left(x_{1}, x_{3}, t\right)
\end{array}\right]
\end{gathered}
$$

where,

$$
\begin{aligned}
x_{1}(t) & :=z_{1}(t), \quad x_{2}(t):=z_{1}(t), \\
x_{3}(t) & :=z_{2}(t), x_{4}(t):=z_{2}(t), \\
x_{5}(t) & :=z_{T}(t), \quad x_{6}(t):=z_{T}(t) .
\end{aligned}
$$

By the use of (4) and (5), the equaitionsc of motion (1), (2) and (3) can be rewritten into state space form as,

$$
x(t)=A x(t)+B_{u} u(t)+B_{w} w(x, t)
$$

where $A \in \mathfrak{R}^{n \times n}$ is the state matrix which is given by,

$$
A=\left[\begin{array}{cccccc}
0 & 1 & 0 & 0 & 0 & 0 \\
a_{21} & a_{22} & a_{23} & a_{24} & a_{25} & a_{26} \\
0 & 0 & 0 & 1 & 0 & 0 \\
a_{41} & a_{42} & a_{43} & a_{44} & a_{45} & a_{46} \\
0 & 0 & 0 & 0 & 0 & 1 \\
a_{61} & a_{62} & a_{63} & a_{64} & a_{65} & a_{66}
\end{array}\right]
$$

with,

$$
\begin{aligned}
& a_{21}=-\omega_{1}^{2}-K_{T} \phi_{1}^{2}, a_{22}=-2 \xi_{1} \omega_{1}-C_{T} \phi_{1}^{2}, \\
& a_{23}=-K_{T} \phi_{1} \phi_{2}, a_{24}=-C_{T} \phi_{1} \phi_{2}, \\
& a_{25}=\phi_{1} K_{T}, a_{26}=\phi_{1} C_{T}, \\
& a_{41}=-K_{T} \phi_{1} \phi_{2}, a_{42}=-C_{T} \phi_{1} \phi_{2}, \\
& a_{43}=-\omega_{2}^{2}-K_{T} \phi_{1}^{2}, a_{44}=-2 \xi_{2} \omega_{2}-C_{T} \phi_{2}^{2}, \\
& a_{45}=\phi_{2} K_{T}, a_{46}=\phi_{2} C_{T}, \\
& a_{61}=\omega_{T}^{2} \phi_{1}, a_{62}=2 \xi_{T} \omega_{T} \phi_{1},
\end{aligned}
$$

$$
\begin{aligned}
& a_{63}=\omega_{T}^{2} \phi_{1}, a_{64}=2 \xi_{T} \omega_{T} \phi_{2}, \\
& a_{65}=-\omega_{T}^{2}, a_{66}=-2 \xi_{T} \omega_{T} .
\end{aligned}
$$

$B_{u} \in \mathfrak{R}^{n \times m}$ is the control input matrix which is given by

$$
B_{u}=\left[\begin{array}{llllll}
0 & -\phi_{1} & 0 & -\phi_{2} & 0 & \frac{1}{m_{T}}
\end{array}\right]^{T}
$$

and $B_{w} \in \mathfrak{R}^{n \times p}$ is the disturbance input matrix which is given by

$$
B_{w}=\left[\begin{array}{llllll}
0 & 1 & 0 & 0 & 0 & 0 \\
0 & 0 & 0 & 1 & 0 & 0
\end{array}\right]^{\mathrm{T}}
$$

\subsection{Disturbance model}

As it is well known that offshore platforms are exposed to the nonlinear self-excited wave forces. In this study, a nonlinear Morison equation is used to calculate horizontal wave force [2]. Let us consider a joint point $p$ on the platform. Unidirectional plane wave forces exerted on this point can be obtained as

$$
\begin{aligned}
F_{p} & =\frac{1}{2} \rho C_{D} A_{p}\left|U_{p x}^{\prime}\right| U_{p x}^{\prime} \\
& +\rho C_{I} B_{p} a_{p x} \\
& -\rho\left|C_{I}-1\right| B_{p} U_{p x} .
\end{aligned}
$$

Here, $F_{p}$ is the wave force vector, $A_{p}$ is the lumped area at point $p, U_{p x}$ is horizontal velocity of water, $U_{p x}^{\prime}$ is horizontal velocity of point $p$, the difference $U_{p x}^{\prime}=U_{p x^{-}}$ $\dot{U}_{p x}$ is the relative velocity of water with respect to point p. $C_{D}$ and $C_{I}$ are drag and inertia coefficients, relatively, $a_{p x}$ is horizontal acceleration of wave on point $p, B_{p}$ is lumped volume on $p, \rho$ water density, and $\ddot{U}_{p x}$ is horizantal acceleration of point $p$. Velocity and acceleration of a horizontal advancing wave are related to wave characteristics and properties of motion area of the wave.

At point $p$, the horizontal velocity is given as,

$$
U_{p x}^{\prime}=E_{p} \cos \left(k x_{p}-\Omega t\right)+U_{o w} \frac{Y_{p}}{h}-U_{p x} .
$$

The horizontal acceleration is expressed as,

$$
a_{p x}=E_{p} \Omega \sin \left(k x_{p}-\Omega t\right)
$$

where,

$$
E_{p}=\frac{\Omega H \cosh \left(k Y_{p}\right)}{2 \sinh (k h)}
$$

$x_{p}$ and $Y_{p}$ are the location of point $p$ with respect to a fixed coordinate axes reference, hence $Y_{p}$ is the height of point $p$ from seabed, $h$ is water depth, $\Omega$ is the frequency of the water, $H$ is the height of water, $\lambda$ is the wavelength, $k=2 \pi / \lambda$ is wave number and $U_{o w}$ is the current velocity of the water surface. The aforementioned self excited hydrodynamic forces $f_{1}, f_{2}$, $f_{3}$ and $f_{4}$ can be computed by the use of Equations (10)(13). 


\section{Optimal state derivative feedback $L Q R$ controller design}

In the last decade, the state derivative feedback has been extensively studied. Design of a full state derivative feedback control with state derivative estimator for acceleration feedback has been presented by Kwak et al. [9]. Abdelaziz and Valasek have developed a procedure to design state derivative feedback controller for pole placement of single input single output linear systems [10]. Then, design of robust pole placement and optimal regulator problems with state derivative feedback has been presented in [11], [12]. Linear Matrix Inequalities (LMIs) based solvability conditions for state derivative feedback has been firstly designed by Assunçao et al. [13]. Faria et al. have extended the problem with regional pole placement [14]. L2 gain state derivative feedback controller design has been formulated via LMIs by Sever and Yazici [15].The proposed $\mathrm{L}_{2}$ gain state derivative feedback control approach has been extended with robustness against polytopic type uncertainties [16]. Despite the fact that LMI based solutions of classical LQR approach is widely used in the literature [17], [18], [19], design of a state derivative feedback LQR via LMIs has not been considered so far as provided in this paper.

In this section, first an optimal state derivative feedback LQR controller synthesis is presented. Then, to compare the proposed state derivative feedback LQR controller, design of a classical LQR controller synthesis is provided.

Consider the linear time-invariant system described by

$$
x(t)=A x(t)+B u(t)
$$

where $x(t) \in \mathfrak{R}^{n}$ is the state vector and $u(t) \in \mathfrak{R}^{m}$ is thecontrol input vector. Our goal is to find an optimal state derivative feedback control in the form of

$$
u(t)=-K x(t)
$$

where $K \in \mathfrak{R}^{m \times n}$ is a controller gain matrix. The closed-loop system is written in the reciprocal state space framework [9] by replacing the (15) into (14) as follows.

$$
x(t)=A^{-1}(I+B K) x(t)
$$

The quadratic cost function is given by

$$
J=\int_{0}^{\infty} x(t)^{\mathrm{T}} Q x(t)+u(t)^{\mathrm{T}} R u(t) d t .
$$

here, $Q \in \mathfrak{R}^{n \times n}$ and $R \in \mathfrak{R}^{m \times m}$ are the performance weight matrices. The following theorem presents a LMI based method to design optimal state derivative feedback LQR controller.

Theorem. For a given values of $Q$ and $R$, asymptotic stability of the reciprocal state space closed-loop system (16) is ensured with a minimum value of the quadratic cost function (17), if there exists a solution for the following optimization problem

$$
\begin{aligned}
& {\left[\begin{array}{ccc}
A^{-1} S+A^{-1} B W & S & W^{\mathrm{T}} \\
+S A^{-\mathrm{T}}+W^{\mathrm{T}} B^{\mathrm{T}} A^{-\mathrm{T}} & & \\
* & -Q^{-1} & 0 \\
* & * & R^{-1}
\end{array}\right]<0} \\
& {\left[\begin{array}{ll}
M & I \\
* & S
\end{array}\right]>0 .}
\end{aligned}
$$

Then, the optimal control law can be calculated as $u(t)=-K x(t)=-W S^{-1} x(t)$.

Proof. By substituting the (15) into (17), the cost function is turned into

$$
J=\int_{0}^{\infty} x(t)^{T}\left(Q+K^{T} R K\right) x(t) d t .
$$

Suppose that a positive definite matrix $P$ exists which satisfies the equation (21) [20]

$$
x(t)^{T}\left(Q+K^{T} R K\right) x(t)=-\frac{d}{d t}\left(x^{T}(t) P x(t)\right) .
$$

By integrating the (21)

$$
\begin{aligned}
& J=-\left.x^{T}(t) P x(t)\right|_{0} ^{\infty}= \\
& -x^{\mathrm{T}}(\infty) \operatorname{Px}(\infty)+x^{T}(0) \operatorname{Px}(0)
\end{aligned}
$$

is obtained. Under the assumption of the closed-loop system (16) is asymptotically stable, the cost function converges to the

$$
J=x(0)^{T} P x(0) .
$$

Hence, the equation (21) can be rewritten as follows

$$
\begin{aligned}
& x(t)^{T}\left(Q+K^{T} R K\right) x(t)= \\
& -\left(x^{T}(t) P x(t)+x^{T}(t) P x(t)\right) .
\end{aligned}
$$

By using the closed-loop system (16) in reciprocal state space framework, (24) is converted to

$$
\begin{aligned}
& x(t)^{T}\left(Q+K^{T} R K\right) x(t)= \\
& -x^{T}(t)\left(\begin{array}{c}
P A^{-1}(I+B K)+ \\
(I+B K)^{T} A^{-T} P
\end{array}\right) x(t) .
\end{aligned}
$$

Design of an optimal state derivative feedback controller problem can be cast to the matrix inequality constraint problem by change of variables. Let us define a new variable $Y=Y^{T}>P$. Then, substituting $Y$ into (25) allows us to write

$$
\begin{aligned}
& Y A^{-1}+A^{-\mathrm{T}} Y+Y A^{-1} B K \\
& +K^{\mathrm{T}} B^{\mathrm{T}} A^{-\mathrm{T}} Y+K^{\mathrm{T}} R K+Q<0 .
\end{aligned}
$$

By applying the Schur complement formula [21], (26) is congruent to 


$$
\left[\begin{array}{ccc}
Y A^{-1}+Y A^{-1} B K+ & & K^{\mathrm{T}} \\
A^{-\mathrm{T}} Y+K^{\mathrm{T}} B^{\mathrm{T}} A^{-\mathrm{T}} Y & I & \\
* & -Q^{-1} & 0 \\
* & * & R^{-1}
\end{array}\right]<0 .(27)
$$

(27) is not in the LMI form yet due to the multiplication of decision variables $Y$ and $K$. Pre and post multiply the (27) by $\operatorname{diag}(S, I, I)$ where $S=S^{T}=Y^{-1}$ and (18) is obtained. Here, $W=K S$ is a modest variable change operation. Recall that the quadratic cost function (23) has to be minimized by optimal state derivative feedback control law (15). Then, a new decision variable $M \in \mathfrak{R}^{c \times c}$ is introduced to set an upper bound on the cost as follows:

$$
M>Y \leftrightarrow\left[\begin{array}{cc}
M & I \\
* & S
\end{array}\right]>0 .
$$

In the light of the results obtained above, the proof is completed.

\subsection{Classical $L Q R$ controller design}

In order to compare the effectiveness of the proposed state derivative feedback LQR controller, a classical LQR controller has been designed in this subsection.

As it is well known that LQR control problem is to find an optimal state feedback control law that minimized the quadratic cost function with the solution of following Algebraic Riccati Equation [22],

$$
S A+A^{\mathrm{T}} S+Q+S B R^{-1} B^{\mathrm{T}} S=0 .
$$

Then, the classical state feedback control law can be obtained as

$$
u_{c}(t)=K_{c} x(t)=-R^{-1} B^{\mathrm{T}} S x(t) .
$$

\section{Numerical examples}

In this section, extensive numbers of simulations are carried out to verify the effectiveness and applicability of the proposed controller to a offshore steel jacket platform subject to nonlinear wave disturbance. The parameters of the considered offshore steel jacket platform having ATMD are taken from [2], [23] and listed in Table 1 . In addition, non-linear self excited wave force $w(x, t)$ have been computed as Appendix A in [2]. All the simulations and computations are employed using Matlab with Simulink.

The matrices $A$ and $B_{u}$ of the modeled offshore steel jacket platform can be written by the use of these system parameters as

$$
\begin{gathered}
A=\left[\begin{array}{ccc}
0 & 1 & 0 \\
-3.3235 & -0.0212 & 0.0184 \\
0 & 0 & 0 \\
0.0184 & 0.0030 & -118.135 \\
0 & 0 & 0 \\
-0.0114 & -0.0019 & 0.0114 \\
0 & 0 & 0 \\
0.0030 & -5.3449 & -0.8819 \\
1 & 0 & 0 \\
-0.1118 & 5.3465 & 0.8822 \\
0 & 0 & 1 \\
0.0019 & -3.3051 & -0.5454
\end{array}\right] \\
B_{u}=\left[\begin{array}{llll}
0 & 0.003445 & 0 & \\
-0.00344628 & 0 & 0.00213
\end{array}\right]^{\mathrm{T}} .
\end{gathered}
$$

Table 1. Parameter values of considered offshore steel jacket platform.

\begin{tabular}{|l|c|}
\hline Parameter & Value \\
\hline Wave Height $(H)$ & $12.19 \mathrm{~m}$ \\
\hline Wave Length $(\lambda)$ & $182.88 \mathrm{~m}$ \\
\hline Depth of Water $(h)$ & $76.2 \mathrm{~m}$ \\
\hline Current Velocity $\left(U_{\text {ow }}\right)$ & $0.122 \mathrm{~m} / \mathrm{s}$ \\
\hline Wave Frequency $(\omega)$ & $1.8 \mathrm{rad} / \mathrm{s}$ \\
\hline Natural Frequency $\left(\omega_{1}\right)$ & $1.818 \mathrm{rad} / \mathrm{s}$ \\
\hline Natural Frequency $\left(\omega_{2}\right)$ & $10.8683 \mathrm{rad} / \mathrm{s}$ \\
\hline Damping Ratio $\left(\xi_{1}\right)$ & 0.005 \\
\hline First Mode Shape $\left(\phi_{1}\right)$ & -0.003445 \\
\hline Second Mode Shape $\left(\phi_{2}\right)$ & 0.00344628 \\
\hline Damping Ratio $\left(\xi_{2}\right)$ & 0.005 \\
\hline Natural Frequency of & $1.8180 \mathrm{rad} / \mathrm{s}$ \\
ATMD $(\omega \mathrm{T})$ & \\
\hline Mass of ATMD $\left(m_{\mathrm{T}}\right)$ & $469.4836 \mathrm{~kg}$ \\
\hline Stiffness of ATMD $\left(K_{\mathrm{T}}\right)$ & 1551.5 \\
\hline Damping of ATMD $\left(\mathrm{C}_{\mathrm{T}}\right)$ & 256 \\
\hline Damping Ratio $\left(\xi_{\mathrm{T}}\right)$ & 0.15 \\
\hline
\end{tabular}

The performance weight matrices which are used in controller design are

$$
\begin{aligned}
& Q=\operatorname{diag}(500,500,500,500,500,500), \\
& R=0.001 .
\end{aligned}
$$

In the light of Theorem, in order to minimize the quadratic cost given by (31), proposed controller is designed. For the solution of the resulting LMIs, Yalmip parser and Sedumi solver are used [24], [25].Thus, the optimal state derivative feedback LQR control law is computed as

$$
\begin{aligned}
u(t) & =-K x(t) \\
& =-10^{3} \times\left[\begin{array}{llll}
1.1282 & -0.3589 & -0.1116 \\
& -0.0110 & 2.0639 & 1.4837
\end{array}\right] x(t) .
\end{aligned}
$$

For brevity from this point onwards we will henceforth denote this controller as SDFLQR. 
In addition to compare the performance of the proposed controller, the classical LQR controller has been designed for given system (14) with the performance weighting matrices (31). The resulted classical LQR control law can be obtained as

$$
\begin{aligned}
& u_{c}(t)=K_{c} x(t) \\
& =10^{3} \times\left[\begin{array}{lll}
-1.3936 & -0.2053 & 2.9085
\end{array}\right. \\
& 0.6227-0.1532-1.5248] x(t) \text {. }
\end{aligned}
$$

For brevity from this point onwards we will henceforth denote this controller as LQR. The displacement responses of the first, second and third floors of the offshore steel jacket platform are shown in Figure 2, Figure 3 and Figure 4, repectively for the controlled and uncontrolled cases against the nonlinear wave forces.

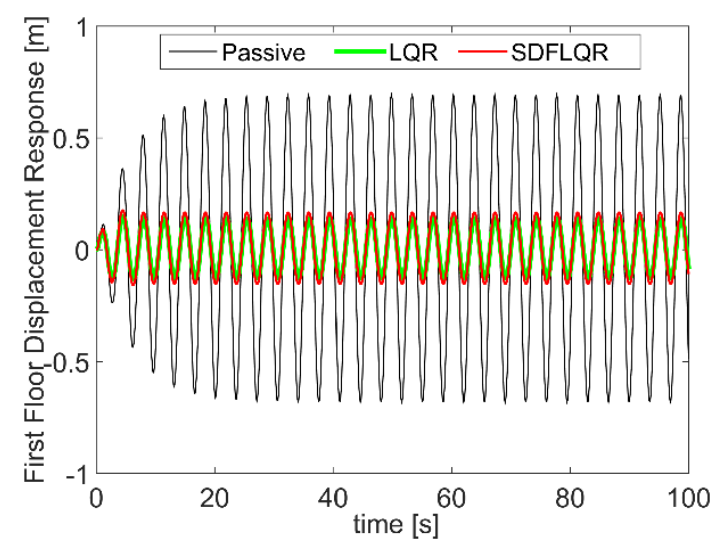

Figure 2. Controlled and uncontrolled displacemet time responses of first floor of offshore steel jacket platform.

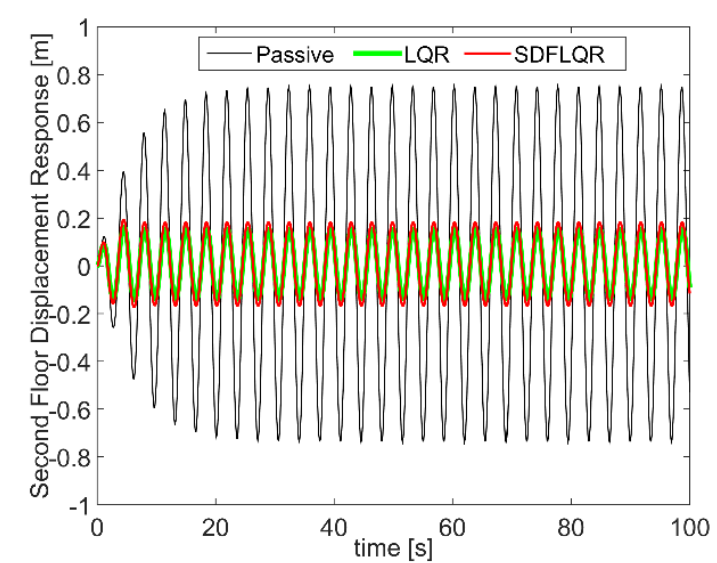

Figure 3. Controlled and uncontrolled displacemet time responses of second floor of offshore steel jacket platform.

As shown in Figure 2, Figure 3 and Figure 4, vibration amplitudes of each floor of the offshore steel jacket platform are suppressed successfully by the use of SDFLQR and classical LQR. On the other hand Figure 5 demonstrates the cahange in control inputs for SDFLQR and classical LQR.

When the response plots of the offshore steel jacket platform with uncontrolled and controlled cases are compared,SDFLQR and classical LQR have very close vibration suppression performance. On the other hand, by taking into account that the state derivative signals are much available to obtain good accuracy, proposed SDFLQR is very promising solution for active vibration control of offshore steel jacket platform having nonlinear self excited wave induced disturbances.

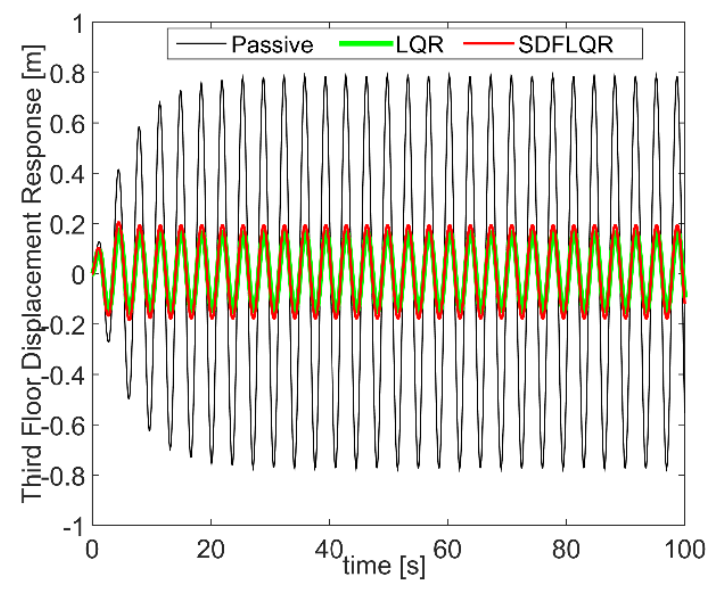

Figure 4. Controlled and uncontrolled displacemet time responses of the third floor of offshore steel jacket platform.

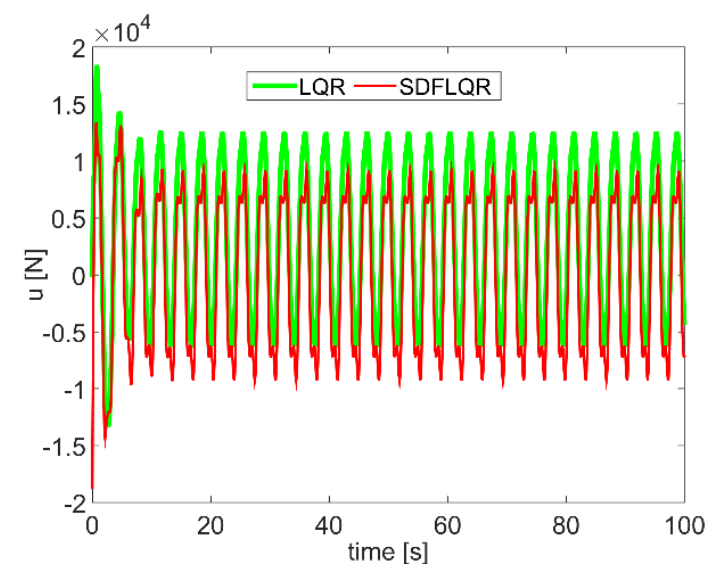

Figure 5. Time history of the applied control force for SDFLQR and LQR.

In this section, the root mean square (RMS) value, which is statistic measure of the magnitude of varying quantity, is employed to investigate the active vibration control performance. RMS analysis method is very useful to evaluate active control performance when the variants are positive and negative [26]. The corresponding RMS values of displacement responses of each floor of the considered offshore steel jacket platform and applied control forces are compared for the both controlled and uncontrolled cases in Table 2 for nonlinear wave disturbance input.

Remark: As can be observed from Table 2, proposed SDFLQR achieves compitable control performance to classical LQR control method. Note that the system response and the control effort is not equally invloved in the quadratic cost functions of both LQR and SDFLQR. The control input is weighted with a state derivative vector as $\dot{x}(t)$ for SDFLQR and weighted 
with a state vector as $x(t)$ for LQR. Therefore, similar choice of $Q$ and $R$, results dissimilar performance objectives for SDFLQR and LQR. In the light of aforementioned discussions, it is natural to have slighlty different vibration attenuation levels. It is noteworthy that previously applied control approaches in the literature are applied state feedback to actively control the steel jacket platform vibrations [4], [5], [6], [7] and [8]. Accessing the displacement information from accelerometer outputs by a double integration, is required to realize state feedback control law. Considering that state derivative signals are much available to obtain good accuracy, proposed SDFLQR design process provides more practically applicable and easily realizable synthesis method and has a great potential for active vibration control of offshore steel jacket platform.

Table 2. Comparison of RMS values of displacement responses of each floor of the offshore steel jacket platform and applied control forces for the both controlled and uncontrolled cases.

\begin{tabular}{lccc}
\hline Displacements & \multicolumn{3}{c}{ RMS Values } \\
\cline { 2 - 4 } $\begin{array}{l}\text { and Applied } \\
\text { Control Force }\end{array}$ & Passive & LQR & SDFLQR \\
\hline First Floor (m) & 0.4589 & 0.0967 & 0.1147 \\
Second Floor (m) & 0.4969 & 0.1056 & 0.1249 \\
Third Floor (m) & 0.5212 & 0.1122 & 0.1322 \\
Control Force (N) & - & $7.6782 \times 10^{3}$ & $7.1554 \times 10^{3}$ \\
\hline
\end{tabular}

\section{Conclusion}

This paper presents an approach for designing state derivative feedback LQR controller to attenuate the vibration occurred in offshore steel jacket platform against the nonlinear wave forces. In controller design, the solvability conditions of the proposed control strategy is presented as LMI constraints on the basis of convex optimization approach. The main importance of this study is to devoloped an easily realizable synthesis method to obtain practically applicable optimal state derivative LQR controller which provides satisfactory control performance. In order to demonstare the effectiveness of the approach, performance of the proposed controller is examined in disturbance attenuation of nonlinear wave force excitations, in an offshore steel jacket paltform having ATMD. Simulation results indicate that the proposed control technique is all effective in reducing vibration amplitudes of each floor and guarantees the closed-loop stability. Vibration attenuation performance of the proposed controller can be improved by employing pole location constraints via LMI regions and $H_{2} / H_{\infty}$ norm conditions. Finally, to cope with the practical problems such as parametric uncertainties and actuator imperfections, expanding the proposed method with the robustness against actuator delay and uncertain parameters might be a significant direction for future work.

\section{Acknowledgments}

The authors would like to thank the Associate Editor and anonymous reviewers for their valuable comments.

\section{References}

[1] Abdel-Rohman, M.(1996). Structural Control of Steel Jacket Platform. Structural Engineering and Mechanics, 4(2), 125-138.

[2] Terro, M.J., Mahmoud, M.S., Abdel-Rohman, M. (1999). Multi-loop Feedback Control of Offshore Steel Jacket Platforms. Computers and Structures, 70(2), 188-202.

[3] Zribi, M., Almutari, N., Abdel-Rohman, M., Terro, M. (2004). Nonlinear and Robust Control Schemes for offshore platforms. Nonlinear Dynamics, 35(1), 61-80.

[4] Wu, H.Z., Bao-lin, Z., Hui, M.A. (2012). Active Non-Fragile Control for Offshore Steel Jacket Platforms. Proceedings of the 31st Chinese Control Conference, 7481-7486, July 25-27, Hefei, China.

[5] Mei , X.Y., Bao-Lin, Z., Jian-Cun, W., Xiefu, J. (2016). Fuzzy $H_{\infty}$ Control for Steel Jacket Platforms with Parameter Uncertainties, Proceedings of the 35st Chinese Control Conference, 3749-3754, July 27-29, Chengdu, China.

[6] Zhang B.L, Ma L., Han Q.L. (2013). Sliding mode $\mathrm{H}_{\infty}$ Control for offshore steel jacket platforms subject to nonlinear self excited wave force and external disturbance. Nonlinear Analysis: Real World Applications, 14(1), 163-178.

[7] Li, H.J., Lan S., Hu, J., Jakubiak, C. (2003). $\mathrm{H}_{2}$ active vibration control for offshore platform subject to wave loading. Journal of Sound and Vibration, 263(4), 709-724.

[8] Zhang B.L., Hu Y.H., Tang, G.Y. (2012). Stabilization control for offshore steel jacket platforms with actuator time-delays. Nonlinear Dynamics, 70(2), 1593-1603.

[9] Kwak S.K., Washington G., Yedavalli R.K. (2002). Acceleration-based vibration control of distributed parameter systems using the "reciprocal state-space framework". Journal of Sound and Vibration, 251(3), 543-557.

[10] Abdelaziz T.H.S. and Valasek M. (2004). Pole placement for SISO linear systems by state derivative feedback. IEE Proceedings-Control Theory and Applications, 151(4), 377-385.

[11] Abdelaziz T.H.S. (2009). Robust pole assignment for linear time-invariant systems using statederivative feedback. Journal of Systems and 
Control Engineering, 223(2), 187-199.

[12] Abdelaziz T.H.S. (2010). Optimal control using derivative feedback for linear systems. Journal of Systems and Control Engineering 224(2), 187202.

[13] Assunçao E, Teixeira MCM, Faria FA et al. (2007). Robust state derivative feedback LMIbased designs for multivariable linear systems. International Journal of Control, 80(8), 12601270 .

[14] Faria FA, Assunçao E, Teixeira MCM et al. (2009). Robust state derivative pole placement LMI based designs for linear systems. International Journal of Control, 82(1), 1-12.

[15] Sever M., Yazici H. (2017) Active Control of Vehicle Suspension System Having Driver Model via $\mathrm{L}_{2}$ Gain State Derivative Feedback Controller. 2017 4th International Conference on Electrical and Electronics Engineering (ICEEE 2017), 215222, April 8-10,Ankara, Turkey,

[16] Yazici H, Sever M. (2017). L2 gain state derivative feedback control of uncertain vehicle suspension systems. Journal of Vibration and Control,In Press.

[17] Aktas A, Sever M, Yazici H. (2016). Gain scheduling LQR control of linear parameter varying overhead crane. In: IEEE 2016 National Conference on Electrical, Electronics and Biomedical Engineering,232-236, Dec 1-3; Bursa, Turkey.

[18] Sever M, Kaya EE, Arslan MS, Yazici H. (2016) Active trailer braking system design with linear matrix inequalities based multi objective robust LQR controller for vehicle-trailer systems. In: IEEE 2016 Intelligent Vehicles Symposium,726731, June 19-22, Gothenburg, Sweeden.

[19] Yazici H, Sever M. (2017). Active control of a non-linear landing gear system having oleo pneumatic shock absorber using robust linear quadratic regulator approach. Proceedings of the Institution of Mechanical Engineers, Part G: Journal of Aerospace Engineering, In Press.

[20] Abdelaziz THS, Valasek M. (2005). State derivative feedback by LQR for linear time invariant systems. IFAC Proceeding Volumes,
38(1), 435-440.

[21] Boyd, S., Ghaoui, L.E., Feron, E., Balakrishnan, V. (1994)Linear Matrix Inequalities in System and Control Theory. Society for Industrial and Applied Mathematics (SIAM), Philadelphia.

[22] Lin, F. (2007).Robust Control Design an Optimal Control Approach. John Willey\&Sons, Hartfordshire.

[23] Bao-Lin, Z., Han, Q.L. (2014). Network-based Modelling and Active Control for Offshore Steel Jacket Platform with TMD Mechanisms. Journal of Sound and Vibrations, 333(25), 6796-6814.

[24] Löfberg, J. (2004) Yalmip: A Toolbox for Modeling and Optimization in MATLAB. Proceedings of the CACSD Conference, Taipei, Taiwan.

[25] Strum, J.F. (1999). Using SeDuMi 1.02 a Matlab for optimization over symmetric cones. Optimization Methods and Software, 11(2), 625653.

[26] Zhao, Y., Sun, W., Gao, H. (2010). Robust control synthesis for seat suspension systems with actuator saturation with time varying input delay. Journal of Sound and Vibration, 329(21), 43354353.

Hakan Yazici received his M.Sc and Ph.D. from Yildiz Technical University, all in Mechanical Engineering, in 2006 and 2011, respectively. He conducted post-doctoral research at Southern Illinois University Edwardsville, USA, from 2011 to 2012. His current research interest includes robust and optimal control of time-delay systems with actuator saturation, stability of functional differential equations with time delays, and active vibration control and its applications.

Mert Sever was born in Istanbul, Turkiye, in 1990. He received his B.Sc from Yildiz Technical University, in Mechanical Engineering, in 2014 and is a Msc. Candidate in Machine Theory and Control Program, in the same university, respectively. $\mathrm{He}$ is currently a Research Assistant at Yildiz Technical University, Department of Mechatronics Engineering. His current research interest includes LMI based robust and optimal control, active vibration control and its applications.

An International Journal of Optimization and Control: Theories \& Applications (http://ijocta.balikesir.edu.tr)

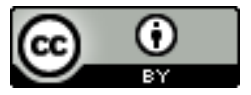

This work is licensed under a Creative Commons Attribution 4.0 International License. The authors retain ownership of the copyright for their article, but they allow anyone to download, reuse, reprint, modify, distribute, and/or copy articles in IJOCTA, so long as the original authors and source are credited. To see the complete license contents, please visit http://creativecommons.org/licenses/by/4.0/. 